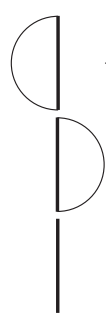

Abstract International collaboration projects in academic work can be considered boundary-crossing projects with learning potential. Contrary to perceiving diversity as a barrier for understanding, we depart from dialogical arguments in perceiving ambiguity and diversity as continuous resources for meaning enrichment. Here, we report a study of an international academic project to gain more insight into how this resource is exploited. Using Bakhtin's theory, negotiation processes are analysed and explained by distinguishing voices stemming from different socio-cultural backgrounds. Project members did not explore fully the voices being expressed in their negotiation processes and therefore did not come to face their differences. We conclude that diversity should neither be seen as an obstacle for understanding, nor be presupposed as a resource for meaning generation. Rather,

diversity should be actively worked on by group members in collaboration, starting by perceiving each other as real 'others' and receiving arguments initially as not understood.

Key Words boundary crossing, collaboration, dialogical processes, discourse analysis, diversity, multivoiced

Sanne Akkerman

Utrecht University, The Netherlands

Wilfried Admiraal

University of Amsterdam, The Netherlands

Robert Jan Simons

Utrecht University, The Netherlands

Theo Niessen

Maastricht University, The Netherlands

\title{
Considering Diversity: Multivoicedness in International Academic Collaboration
}

Collaboration in work settings allows professionals to come into contact with ideas and approaches of other professionals, enabling them to reflect on their own ideas and approaches and to consider alternative ideas and approaches. More generally put, being in dialogue with others entails the transcendence of private worlds (Wertsch, 1985). Advancement of new ideas, that is, conceptual 
development, is a core feature of academic work, the field that is addressed in this study. As Star and Griesemer (1989) have noted, scientific work is heterogeneous, requiring many different actors and viewpoints as well as requiring collaboration. In a previous study (Akkerman, Admiraal, \& Simons, 2006), we found that academics are particularly engaged in and motivated by international collaboration projects. As Knorr Cetina (1999) has pointed out, epistemic cultures, shaped by affinity, necessity and historical coincidence, determine how people know and what they know. As a crossing between epistemic cultures, these international collaboration projects, we argue, require academics to intensively share and create what they know, and so develop their professional expertise as well as enrich the various research communities that they are representing. This study describes an international academic collaboration project, partly financed by the European Commission, and considers how diversity in the project group in terms of multiple different viewpoints comes to the fore in the collaborative work in the project.

\section{Collaboration and Diversity}

Throughout the social sciences it has been argued that boundary crossing between groups or difference and diversity within groups is a resource for meaning generation (e.g. Engeström, Engeström, \& Kärkäinen, 1995; Granovetter, 1973; Gutiérrez, Baquedano-López, \& Tejeda, 1999; Homan, 2001; Ryan, Cott, \& Robertson, 1997; Wenger, 1998). What is also frequently emphasized is that the use of multiple different lenses to approach an object of investigation allows groups to deal with complex cases and problems (Derry, Adams DuRussel, \& O'Donnell, 1998; Yoo \& Kanawattanachai, 2001). Although diversity is argued to be a potential resource for new understandings, a general argument in many studies on collaboration is that diversity is also that which constrains mutual understanding and learning from each other (e.g. Marks, Burke, Sabella, \& Zaccaro, 2002; Mathieu, Heffner, Goodwin, Salas, \& Cannon-Bowers, 2000; Mohammed \& Ringseis, 2001). Diverse viewpoints are then considered a barrier. Gurevitch (1988) comments that:

The central assumption of phenomenological and related approaches holds that the possibility of mutual understanding and communication in interpersonal relations is contingent on the supposition of the sameness of self and other. Under this assumption, the otherness of the other is conceived as an obstacle to achieving mutual understanding. (p. 1179)

Difference between people's viewpoints, or 'otherness', is perceived as something that should be overcome or taken away. In a similar mode, 
Matusov (1996) noted that intersubjectivity is often viewed as a process in joint activity, progressing 'from heterogeneity to increasing symmetry among the individuals' perspectives and prolepses' (p. 26). These two authors argue against an understanding of diversity as an obstacle. In doing so, they criticize the basic and commonly held presupposition that communication is but a transmission process. As described and also criticized by Wertsch and Toma (1995), this common view on communication assumes that any text message represents a meaningful notion for each speaker, which is to be 'correctly' transmitted to the listener through a process of encoding, transmitting, decoding and storing. In line with the theory of Lotman $(1990,1994)$, Wertsch and Toma noted that texts have two functions, one univocal and the other dialogic. Whereas the univocal function involves conveying meaning adequately, the dialogic function involves generating new meanings. Through examples taken from classroom discourse, Wertsch and Toma portrayed how certain interactions can be dominated by either one of these two functions. To illustrate the dialogic function, they show how pupils at many points take the words and comments of others by reformulating them in their own words to respond to (reject, incorporate or take further) what has been said by the other. According to them, this is a good example of how one speaker's voice can come into contact with and 'interanimate' the voices of others in a dialogic encounter. Building on Lotman's theory, their study points out that the inherent open-ended nature of language, its unfinalized meaning, or the 'noise in the channel of communication' (Alexandrov, 2000, p. 12) should be seen as resource for something new. Ambiguity of language, words meaning different things to different people, is to be acknowledged as contributing to the creative aspect of communication and of collaboration.

Following these arguments on dialogicality, in our view diversity in collaboration not only should be considered as an initial resource that needs to be overcome by 'sameness' between people's viewpoints, but it can also be considered to be a continuous resource for generating new meaning. Yet we still need to understand how this continuous generative function of diversity is at work in collaborative boundary-crossing groups, and come to understand how diversity comes to the fore in collaborative work. The current study questions this in the context of an academic European collaboration project.

\section{Collaboration and Multivoicedness}

Actions and interactions in a collaboration project do not stand on their own in time and place, and much of it can to a large extent be 
understood only within a broader perspective. Project members can be regarded as individuals with unique life histories that inform their professional identities. The specific activities and the socio-cultural contexts in which an academic is and has been working developed his views and his way of behaving. Just as the participants have their unique life histories, the project group itself is also situated in certain contexts. How the group originated, what the previous relations between the participants are and who are possible stakeholders are all elements that inform how the project is shaped and taken up by the participants. As noted by Hermans and Kempen (1993): 'This means that the microcontext of concrete dialogical relationships cannot be understood without some concept of macroframes (organizational and ethnographic context)' (p. 73). In a collaboration project, the identity of the professionals and the actions they undertake partly lie in a broader social, cultural and historical world. Extending the scope with which we look at collaboration projects beyond the immediate activities of the group is therefore a more fundamental and necessary response to the critical socio-genetic question posed by Valsiner and Van der Veer (2000): 'How to construe persons as being social without abandoning their obvious personal autonomy, separateness from any social unit (group, crowd, community), while being members of such units?' (p. 6). In Figure 1, a socio-cultural scope on collaboration projects is pictured. The inner circle (circle 2) represents the collaborative activity involving a group of participants (p1, p2, p3, ...). At the same time each of these participants is a unique individual (i1, i2, i3, ...) with a unique history and is active in other social settings (circle 1), and informed by a broader socio-cultural context (circle 3).

In order to account for the broader socio-cultural contexts of the group and of the participants we need to know how specific historical, cultural and institutional settings are interwoven with various forms of actions and how these are entwined in the individual mind. Some concepts within dialogical perspectives enable us to provide initial answers to these questions.

Bakhtin (1981, 1986; Morris, 1994; Wertsch, 1991) introduced the relevant concept of voice. The three circles in Figure 1 can be seen as three levels of voices. According to Bakhtin, an utterance is always produced by a certain voice, a speaking personality with a specific viewpoint. For example, a person or a group may always advance a focus on products and outcomes or advance a certain theoretical viewpoint, continuously considering the material in specific theoretical terms. Bakhtin also pointed out that an utterance is always reacting to former and anticipating future utterances. It reflects not only the voice 


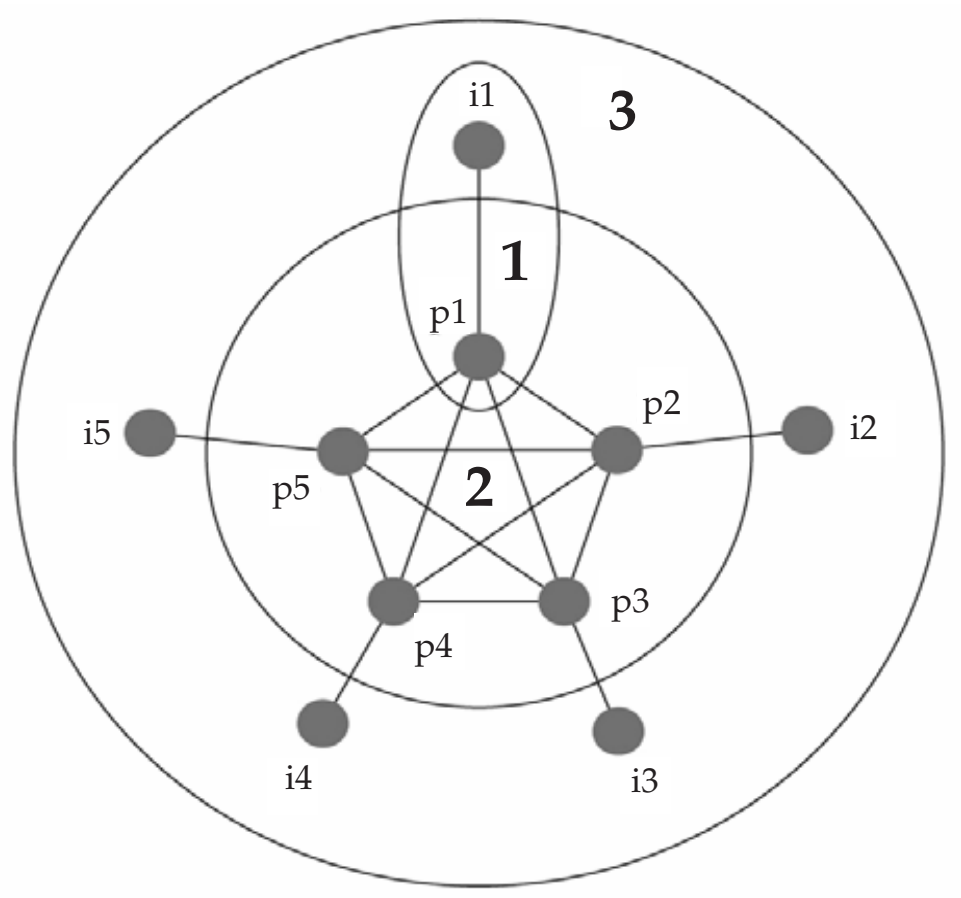

i1-i5: individuals; $\mathrm{p} 1-\mathrm{p} 5$ : participants in group circle 1: unique life history of participants circle 2: collaborative activity circle 3: socio-cultural context

Figure 1. Socio-cultural scope of a collaboration group

that produces it, but also the voices to which it is addressed. People talk differently to a child than to an adult. People talk differently to a friend than to an enemy. They consider the point of view of the listener. As such, an utterance reflects multiple voices. One specific voice may easily be confronted with or opposed to another voice. For example, one person's focus on products and outcomes can become opposed with another person's tendency to work creatively without having in mind yet what products has to result from it. Remarks or actions of the second person can be read and taken by the first product-oriented person as having no direction and therefore no relevance to the collaborative work at hand. In that sense the diverse voices of the project members also react and inform each other. This idea is what Bakhtin 
refers to by the concept of multivoicedness or dialogicality. This also makes clear that an utterance can only be understood within its conversational context. Although individual utterances are unique and situated, Bakhtin (1981) also observed that there are specific 'social languages', that is, discourses related to specific strata of society (such as, for example, professional, age, group) within a given social system at a given time, as can be seen in dialects or professional jargons. These inform the voices of individuals, since, as noted by Hermans and Kempen (1993), 'the individual speaks the words of the group, social class, or society to which the individual belongs and reflects the unity of the group, class or society' (p. 114). For example, a person's focus on products and outcomes can be well related to his or her department or society with a history of emphasizing performance in terms of products that one delivers. Specific voices, being invoked and informed as responses in the conversational and collaborative situation at hand, are thus also informed by a broader socio-cultural context with a particular history. Such social languages correspond to what Bakhtin called speech genres. These refer to types of utterances produced by types of voices. Speech genres can be recognized by typical situations of speech communication, by typical themes and meanings of words that are addressed. Advancing a certain theoretical viewpoint involves talking in terms of that theory, although it simultaneously depends on how the specific person understands that theory, what he or she places within that category.

To refer to persons as being social without abandoning their obvious personal autonomy (Valsiner \& Van der Veer, 2000), we want to briefly refer to the dialogical self theory (Hermans, 2001; Hermans \& Kempen, 1993), which extends Bakhtin's theories into a dialogical understanding of the human mind. This theory describes how the self can be understood as a dialogical self, comprised of a dynamic multiplicity of relatively autonomous I-positions through which a person fluctuates, being informed by historical, cultural and institutional experiences and social relationships. Consequently, a person can have different and even conflicting positions towards the same issue because he or she may use different voices. However, these positions cohere in a Self through their dialogical relations: 'The Self can be seen as a synthesizing activity, that is, as a continuous attempt to make the self a whole, despite the existence of parts that try to maintain or even to increase their relative autonomy' (Hermans \& Kempen, 1993, p. 93). In sum, a person can be perceived as an individual moving through different social environments as a transcendent self, historically continuous and unitary, but who lives in moments as an acting 
participant within such specific social environments. As a participant in a collaborative project, one brings in particular voices that are informed by broader socio-cultural worlds. In this study, we will describe how in a boundary-crossing project different voices come to the fore in the negotiation processes of conceptual material. As such we will demonstrate how diverse socio-cultural worlds are brought into the collaborative work and question how diversity carries potential for generating meaning.

\section{The Study of an International Collaboration Project}

The case being presented here consists of an academic European project within the educational sciences. This project was funded by the European Commission. Five different institutes, or 'partners', from Italy, the Netherlands, Denmark, Scotland and Spain were represented, each by two or more participants. They collaborated for two years, with the general aim of formulating advice for educational policy for facilitating teaching e-learning in secondary education. The motivation for the project was to support educational innovation in Europe by addressing the pioneer teachers as agents of innovation and through the creation of facilitative tools for these pioneer teachers. These pioneer teachers were seen as agents of educational innovation and specifically involved in the use of ICT tools in education.

The project was initiated and designed by the Italian partners, who also acted as project leader during the project. There were both national as well as international activities. By use of an electronic work environment all participants coordinated and managed their activities and posted achieved results in the form of documents, or presentations. The project group organized seven meetings with the partners. These meetings lasted for about three days each. The following are the names of the different participants of each country, as they will be referred to in the analysis: ${ }^{1}$

- Italian partners (project leading partners): Carlo (project leader), Emanuela, Felice.

- Scottish partners: Samuel, James, Victor.

- Danish partners: Hans, Astrid, Ingrid.

- Dutch partners: Peter, Ellen.

- Spanish partners: Rosita, Candela, Frida.

- Other related participants: Alison (the coordinator in the European Commission), Carl, and Sigmund (international evaluators of the project). 


\section{Methodological Approach}

The study of this case fits with an ethnographical approach in the sense that it attempts to grasp and characterize the meaning-making process of a social group through a process of observation (Creswell, 1998; Spradley, 1980). According to Geertz (1973), the understanding of a people's culture through ethnography 'exposes their normalness without reducing their particularity' (p. 14).

As is argued by Guba (1981), studies that are based on a naturalistic instead of a rationalistic paradigm require different means for judging the reliability and validity of those studies. ${ }^{2}$ In order to check the current study, we conducted an audit. In an audit (Akkerman, Admiraal, Brekelmans, \& Oost, in press; Halpern, 1983) a second researcher reflects on the data-gathering and analytical processes that lead to specific results and conclusions, and questions to what extent the analytical steps undertaken in the study make sense and are acceptable in relation to the methodological standards of the social sciences, and to the theoretical and methodological approach that are claimed by the author. The auditor concluded that the overall quality of this research was satisfying in terms of reliability and validity. ${ }^{3}$

\section{Data Sources}

To obtain a clear, textured perspective of the European collaboration project we included many different data resources available at the site. First, all messages and documents exchanged by the project members in the electronic environment were stored. Second, four of the project meetings were videotaped and observed. During the observations, notes were made about any interactions and actions that appeared critical to the researcher in terms of negotiating particular understandings. The first author wrote additional notes about the general topics that were discussed during coffee breaks and lunches, possible relevant aspects for the analysis, and her role as researcher during the project meeting. Also, during the fourth and the fifth meeting and the period in between, twelve of the international participants were interviewed. One project member was not interviewed, since she argued that her national colleague would inform us about the project in a similar way as she would do. The interviews addressed the following topics: the work division and perceived roles in their national group (partner); the role of this partner group in the project; the origin of the project; the partner group's general view on the central concepts of the project; their view on collaboration in general; and their perception of the internal national collaboration and the international collaboration (between the partners within the project) to date. The 
project members were also asked to elaborate the development of their views. These interviews allowed us to gather background information about the project members and about the project as such. The reflections and stories of the project members allowed us to reveal the specific voices and also how these were informed by specific sociocultural contexts.

\section{Analysis}

Given the particular context of the project, we looked at the different voices and their role in the negotiation and development of the central conceptual products. The performed discourse analysis based on Bakhtinian ideas about language (Wetherell, Taylor, \& Yates, 2001) has the advantage of looking at the broader social context of the project without making ambiguous global arguments and also avoids analysing only some fragments (Engeström, 1999).

The first step of the analysis aimed to discover 'voices'. The identification of voices occurred by analysing the interviews for descriptions in which a certain viewpoint was attributed to oneself, to others, or to (sub)groups. One could see such descriptions as acts of 'positioning' (Davies \& Harré, 2001) in which one typifies oneself or others. This can include, for example, a positive voice, a specific theoretical voice or a project leader voice. Although these three examples seem to be of a different kind, all of these can be considered to point to a particular viewpoint or subjectivity, reflecting a conceptual horizon and a certain intention. As such, they involve both cognition and motivation. The results of analysing the interviews showed that the project participants attributed more or less the same voices to themselves and each other, thus recognizing similar subjectivities, but they described these in their own terms and with their own associations. The instances in which these voices were described as linked to other groups, departments, institutions or societies to which the particular person belongs were perceived in the analysis as explicit descriptions of social languages informing these voices. This first step resulted in a list of voices at work in the current project, linked to parts of the original texts in which these subjectivities were described.

In the second step of our analysis, we performed the actual analysis of the group activity, focusing on the negotiation processes related to the two central topics in the project. To depict the negotiation processes, we searched for expressions of (mis)understanding and of (dis)agreement. We did so because these acts reveal if and how the multiplicity and diversity of positions towards the object at hand are being considered. With these critical moments of negotiation as anchor 
points, a description was written of what the project group did and said over time regarding the two central topics. ${ }^{4}$

In the third step of our analysis, the positions of the project members within these discussions were explored and interpreted based on the discourse itself and based on those specific voices that were identified in the interviews.

The last step of the analysis concerned a search for certain patterns or tendencies with regard to how diverse voices come into play in the negotiation and development of conceptual material.

\section{Results: Understanding the Pioneer Teacher}

There were two conceptual topics in the project that appeared to be central for how the project group constructed their understanding of the object, the pioneer teacher. These topics were the so-called 'matrix' and the 'syllabus'. By working on the matrix in the form of a document, the project group aimed to define the pioneer teacher and his or her characteristics. This need for defining the pioneer teacher was decided in the kick-off meeting of the project. Samuel, one of the two Scottish members, proposed to write an initial document. In this document he gave an outline of the competences and skills of a pioneer teacher. This document came to be called the 'matrix', and was one of the conceptual products that was developed and discussed extensively throughout the project.

The second topic of the project, planned for the first couple of months, was aimed at the so-called 'syllabus', entailing a 'common European curriculum and model of accreditation concerning ICT skills in education'. This syllabus was a document that needed to describe project members' idea of how to support the development of the pioneer teacher and was also developed and discussed extensively during the project.

These two topics, the matrix and the syllabus, both needed to capture project members' understanding of a pioneer teacher, and were as such considered as the basic European framework within the project. The discussion of these two topics concerned eight different issues that were objects for discussion. The discourse analysis of these issues showed within six of these issues how several specific voices came to the fore during the negotiation and development of the conceptual work. The following two sections portray two threads of discussion, each concerned with one of the eight issues. To avoid being too extensive, we will not present the whole discourse description, but only our analytic interpretation of it. As we will show, the analytic 
reflections point out how the diverse understandings relate to how people in the interviews pointed to specific voices of themselves and of others. The two analytic descriptions of the next section are intended to illustrate the overall conclusions that we draw about the specific processes in which diverse voices are at play in this project. Where useful for illustration purposes, we include some quotes from the actual discourse description..$^{5}$ The first analytic description considers the discussions of 'the understanding of pedagogy'. The second analytic description concerns the syllabus, and represents the discussion of 'the format of the syllabus'.

\section{Discussing a Pedagogical Perspective}

Discussing the matrix, the project members repeatedly talked about incorporating a pedagogical perspective on ICT. This idea refers to the notion that using ICT in education is not solely some technical issue, requiring the pioneer teacher to have specific ICT skills, but rather something that is connected to a basic pedagogical idea of what education is and what it should be. The pedagogical perspective and focus on ICT, as opposed to a technological focus, seemed to characterize most of the individual participants' way of approaching ICT. Moreover, this idea was mentioned in the meetings several times as being part of the project's identity. Despite advancing a pedagogical focus collaboratively, there were some discussions about members' understanding of pedagogy, and people seemed to have different ways of referring to specific pedagogical approaches. The Spanish and the Dutch partners noted several times that it is important to mention specifically contemporary approaches. The Spanish partners especially were very worried about what the other partners meant by pedagogy. Among other moments, Rosita expressed these in the second meeting, when she started commenting on the matrix:

... if we go to the matrix, because one of the things was to refine the matrix. I think we have sent some comments about the matrix. It is one on the matrix that especially worries us. For instance, you come here to the category definition 'pedagogical implementation of ICT: can practise and demonstrate effective pedagogical implementation'. What does this mean to you? Because maybe ... eh there are effective pedagogical implementations that are not educational for me, so to speak, you know.... But most of this software is highly behavioural, behaviourist. ... For me it is apparent that teachers not only use the computer, but use the computer in a very innovative, updated, progressive way. I would like to know your views about that.

Victor says it depends entirely on the activities and the environment if ICT software is supportive or not and adds: 
Victor: $\quad \ldots$ and it is the educational processes which [name of this project] is focused on, more than anything.

Rosita: I think it is very important to also discuss our views about pedagogy. ... For me it is very important to share this very basic educational background.

Carlo: Yes, but this project is about innovation.

Rosita: Yes, but many times when people present educational innovation, and when you scratch a little bit, it is not educational innovation. Then for me it is not interesting in this way. And I am sure this project is not like that. But I want to make sure that it is not.

Samuel: I think that's a very good point. And that's one of the reasons the matrix is evolving. Because in a sense your input there was very significant. I am still building that into it. That's absolutely right. The way in which approaches to learning themselves are being changed.

Following this, Samuel distributed a slightly updated version of the matrix in which he had not yet built in the comments of the Spanish partners.

As in the exchange above, the common reaction of the other partners on the Spanish worries is that they do share a pedagogical perspective on ICT and also agree with understanding pedagogy in terms of contemporary approaches, referring to, for example, 'educational innovation'. However, the issue of how to refer to pedagogy is raised again and again throughout the collaboration by mainly the Dutch and the Spanish partners. For example, the Spanish partners reacted to a specific version of the syllabus, writing that the content of the section learning models and theories actually did not refer to learning models and theories. They proposed referring to a constructivist approach and other contemporary approaches. In response, Astrid changed the name of the section into 'ICT and learning', and thereby did not change the content of the section. This appears to miss the point that the Spanish partners were making. Also one of the Dutch participants, Ellen, expressed her dissatisfaction with the last format of the syllabus made by Carlo. According to her, this syllabus was much more focused on the technical instead of the pedagogical side. As these and other actions indicate, it is likely that the project members use different understandings of pedagogy and the pedagogical focus of ICT.

When looking at the complete process and given the voices identified in the interviews, we can make sense of what is being negotiated. With respect to the pedagogical view of the Spanish partners, Ellen mentioned in her interview that the Spanish advanced a so-called 'critical' view on education. Ellen described this view of the Spanish 
partners as politically correct and connected it to a feminist way of thinking, but also as an approach that is more demand-oriented instead of supply-driven, and that is facilitative and a more free approach. The three Spanish participants themselves also mentioned in their interviews that they were advancing a critical view on education and said that this view was different from the other partners. They described their 'subjectivity' as more theoretical and focused on interaction and on shared responsibility with teachers. They elaborated on how this relates to specific academic literature and to the larger academic group in which they were working within their department. This explicates how what they voice is socio-culturally informed by a particular broader context, and thus expresses a certain social language. According to them the view of the other partners was opposed to theirs and was more focused on technology and producing courses, and also incorporated a more rational top-down approach with a perception of teachers as students. The specific voice of the Spanish partners, as they described it themselves, pointed out the logic and reasoning within their actions and their understandings regarding pedagogy, as well as their worries about how the other partners understood pedagogy. Although the other partners said they agreed with this pedagogical perspective on ICT and the contemporary approaches, they still had different understandings of pedagogy, yet did not discuss what they had in mind. As also noted at some points in the observation notes of the project meetings and as the videotapes show, at several points Rosita expresses the specific Spanish view on pedagogy according to the voice of their group and department, referring to her group as being 'postmodern' and arguing that the project should also be more postmodern.

Comparable to the Spanish partners, the array of comments made by the Dutch project members about pedagogy also express a certain voice, as became clear in their interviews. Ellen noted that they had a community perspective, just like the Italians. Peter described them as having an interest in and focus on didactics while the other partners had a different perspective:

The others are a bit more on the organizational level, from the perspective of the organization. School organization, institution. They look on a more general level to the project than we do, I think. So, that is already a difference in perspective that I observed. That we are more on the didactics and consider that important. This view is related with the persons who do it, Ellen and I think it is important, so that is personal, and additionally it is because we as a Centre [the institution of the Dutch partners] think it is important. 
The pedagogical ideas they bring to the fore in the project appear to be related to and informed by the focus of the workplace in their own department, which points to how their voice expresses a social language. Among other moments, this voice comes into play when they take up the task of writing about pedagogy in the matrix. Peter also described that they as Dutch partners focus on authentic learning as opposed to a focus on traditional guided learning. He relates that to their interest in organizing workshops and active learning and connects it to the ideas, words and pedagogical conception of the Spanish partners. According to him, the traditional guided learning approach is a preference of the Italian and Danish partners. His description makes clear what voice they are expressing by their comments and by their attempt to elaborate more on the pedagogy in the products that the project group was producing.

In the discussion about pedagogy, Astrid reacted to the Spanish and Dutch comments by preferring a more concrete syllabus and she proposed having more general introductions about this pedagogy and not including specific approaches to the syllabus itself. The interviews made clear how this response is in line with a specific voice that the Danish partners express, which differs from that of the other partners. In the interviews with five project members, there is a reference to a theoretical versus a practical voice. All of these five project members describe the Danish partners, and sometimes Astrid in particular, as advancing a practical voice. While others use the term 'practical', Astrid herself described how they as the Danish partners are always 'addressing the practitioner level', connecting that to their continuous consideration of the conceptual products to be practically useful for the teachers for whom they are being made. In her words it is not about being practical, but about being more closely related to the field of practice. She also said that personally she had no interest at all in the theoretical level, opposing the theoretical to her interest in the practitioner. She continued to describe how this focus on practitioners was informed by the Danish partners' departmental and national context:

We have this board of interest parties that could be unions, teacher's organizations, the Ministry of Education. All the Danish bodies that are involved in that educational area. It is their philosophy that we are expressing. So it is very non-academic and very much addressing the practitioner level.

Something particular in the context of this collaborative activity is thus argued by the Danish project member as reflecting the words of their department and their country. 
What is interesting is that the other project members in their interviews described the Danish partners as not providing any theoretical input, and, in opposition to that, they position themselves as project partners on a more theoretical level. Astrid herself also opposes the Danish parrner's practitioner level to the theoretical level of the other partners. As an example of the others being more theoretical, she referred to an episode where James came to a meeting with pages of literature references and an episode in which Simon mentioned academic names and theories.

The specific voice of the Danish partners came into play in the discussions about pedagogy, where Astrid proposed making concrete products and rejected the Dutch and the Spanish proposals to elaborate on pedagogical notions. The Danish partners did not agree with the pedagogical notions since, in line with the voice they were expressing, these notions could be considered as academic and theoretical elaborations. So, at the same time as the Dutch and Spanish partners were emphasizing particular pedagogical ideas in line with the specific voices connected to their institutions, the response of the Danish partners was in line with a focus on the usability of the product, expressing the voice of the field of practice for which the product was being made.

It is possible to imagine that the Danish partners did not at all disagree with the pedagogical notions as such, but that it only appears that way because the partners at that moment expressed a particular voice. Whether or not this is the case is left unexplored by the project partners. Within the appearance of a disagreement, the project members express specific voices, but do not discuss why the other disagrees. Nor do they explain to the other the rationale behind their own comments.

We saw how the different ideas that partners bring to the fore relate to the professional identities of the project members and the diverse social, cultural and historical contexts in which they work. Remarkably, though, the different ideas brought to the fore are never discussed in the light of these diverse contexts, and both agreements and disagreements are left unexplored until the end. Despite this, the project members continue to make all kinds of changes and decisions regarding the conceptual work, and the product seems to absorb more and more 'unfinished sentences' of the multiple voices involved. As a result, the discussions with the multiple voices involved come to sound like a cacophony. 


\section{Discussing the Format of the Syllabus}

The other aim of the project was to create a so-called 'syllabus', which refers to a curriculum to support the development of the pioneer teacher. This aim was first discussed generally by the partners at the kick-off meeting of the project. Before the second meeting, they started working with an Italian version of a syllabus that was provided by Carlo as a potential example of the European syllabus they strived for. In the meeting the project group initiated a document reflecting the European syllabus. This document was discussed and changed many times during the project. During the project activities, the format changed from a European Syllabus, into a Curriculum, into a Curricular Framework, back into a European Syllabus again. These format changes reflect crucial changes in the way the project group perceived the nature of the syllabus. As we will now describe, these changes can be understood in the light of specific voices being expressed during the discussions.

The group started off with the aim of creating a European Syllabus. After the group agreed on a common format in the second meeting they called this syllabus a 'European Curriculum' or just 'curriculum'. With this, they referred to a detailed curriculum for pioneer teachers that would be useful for the whole of Europe. They continued working on specific parts of this curriculum. Such a product allowed the group to express the voice that in the interviews was described as the European level' in this project.

However, during the fifth meeting, Carlo explained to the others that it was difficult to create one and the same curriculum for pioneer teachers for all the partners' countries. This difficulty expressed by Carlo connects to what some participants described in their interviews as a general tension between the national and the European level that they wanted to address. On the one hand, the project group advanced a 'European-level voice', expressing the motive to create something shared in Europe, and useful for the whole project group and the countries that they represented. On the other hand, there was a 'national level voice', expressing all the specific national needs and perspectives that each partner group brought to the fore. Addressing the national level, however, made it hard to create something shared on the European level. In the words of one Scottish project member: 'Thinking of the full consortium: countries are very different, have very different needs. Then I think the most difficult idea is to really convince ourselves that this project can have an added European value.'

The tension between these voices seems to have informed their decision to stop working on a European Curriculum. Instead of 
working on this European Curriculum, they chose to create what they called a 'Curricular Framework'. This framework prevented the need to write down a detailed European curriculum. With this framework they attempted still to represent a sort of shared European basis, and simultaneously to allow the partners to adopt it in the way that best suited the local circumstances of their countries. This framework illustrates the creation of a multivoiced product in which both the European and the national level voice is accounted for.

Nonetheless, this dialogical relation was disturbed near the end of the project when the European Commission as constituent became a stronger party in the discussion. In his interview, Hans briefly referred to the European Commission as a stakeholder that wanted value for the money invested in the project. As such, the European Commission formed a particular voice that came to play a role in the project. During the discussions, this voice was mostly expressed by Carlo, who, as project leader, emphasized many times the importance of doing what they had promised to the European Commission. He argued that they had promised to deliver a European Curriculum, and that they needed to explain to the Commission why they had failed to do so. This episode shows how Carlo tried to express multiple voices at the same time, illustrating the concept of the dialogical self (Hermans, 2001), as we described in our theoretical introduction. As a person, one can take in multiple positions that together can come to constitute the Self, although these positions can conflict with each other. As a project leader, in addition to the European level, Carlo attempted to address the national level that the project group had been arguing for, as well as to express what the European Commission as a stakeholder was aiming for. However, there seems to be a conflict between the diverse concerns (voices) involved. Consequently, bringing in the voice of the European Commission reinvigorated the discussion about the national-level voice and the European-level voice. According to Carlo, the European Commission expected a detailed curriculum that was useful for the whole of Europe. Instead, the project group decided to create only a framework, leaving open the specific curriculum content to be filled according to the local circumstances of the diverse countries. After heavy discussions, the group decided to solve this question by explaining to the Commission in a basic document the change from a European Curriculum to a Curricular Framework. Although this proposal seemed to solve the conflict with the European Commission voice, Carlo referred to other arguments by the Commission about the current 'European Curricular Framework' being too academic and difficult to understand and use. Besides his 
proposal to create a booklet as a more practical document, he came up with a completely new syllabus. This new syllabus was much like the one that was proposed by him at the beginning of the project. Because of time pressure and the need to answer some questions from the Commission, the Spanish and the Dutch partners consented to send this new version, thereby legitimating this new format and the voice of the Commission that this format addresses. The Scottish partners wrote some fundamental comments, and it is clear that the Scots did not agree to send it. In the meeting that followed, Carlo explained why he thought it was necessary to send a new syllabus to Alison, who represented the European Commission. Samuel gave a counterargument that this project is a developmental project, rather than a research project and that it is not about producing deliverables, but about expressing what we have learned along the way'. This remark reflects a different understanding of the relation between the project group and the Commission as constituent than that Carlo implied. Whereas Samuel stressed that the aim of the project was to develop some 'good' products regardless of the initial project plan, Carlo's actions stressed that the project's aim was to deliver the products that the group promised in their agreement with the Commission. Although it does not seem to be a resolved issue, the minutes of the sixth meeting report that the partners agreed on the format of the syllabus as proposed by Carlo. Hans also questioned whether the content of the syllabus was discussed at all during the meeting, as is suggested by the minutes. The discussion in the sixth meeting represents a negotiation with the voice of the Commission, and did not address the specific content of the syllabus. Despite the two new versions that Carlo made in the end, it seems there were still some previous fundamental comments and questions from Samuel and Hans that were left open.

The tensions between Carlo's actions and suggestions and the ideas and suggestions of some of the other project members seem to relate to how Carlo's role was perceived by other project members. In six of the interviews with the project members he was described as the project leader and initiator of the ideas. We recognized their description of Carlo's role during the observed meetings in several instances when he started elaborating on the large picture of the project and what it was aiming at. He had written the project plan together with the other two Italian project members, and one project member argued that therefore Carlo had ownership of the project and understood the whole concept. According to this project member, the partners did not have complete ownership and understanding of the project, since they 
were not engaged in writing the project plan. Additionally this project member made the following remarks quite strongly:

I think the fact that we were not involved from the very beginning is a problem, because it meant that we didn't quite share the whole concept the same way that Carlo did. And I think it has become clear that Carlo has a concept of this which none of us share; we all see it slightly different from the way he does.

One of the Italian project members noted something similar, but put it into the perspective of their country, and pointed to the social language that they were expressing:

... the original idea [of this project] comes from the Italian situation. In Italy there is a strong need for this community of varied teachers. Actually there is a strong need for innovation and schools.... This idea of creating a community of pioneer teachers comes from this need and in recent years we did other projects dealing with teachers trainings on-line. National projects. So it came to our mind more and more, stronger and stronger, the idea that an added value for this community could be the European level. So being not only a national community but also above all a European community. Because we know that in Europe other countries like Denmark, Holland, are expert in this field. They have already done much of the work we need.

Two other project members referred to Carlo's way of approaching the other partners. On the one hand, he seemed to have a bottom-up approach whereby the ideas had to come from the people in the group. On the other hand, he used a top-down approach in the sense that he had specific objectives in mind and steered the discussion in that direction. As opposed to the comments of these project members, one Italian project member mentioned in her interview that the other partners themselves showed a passive attitude and that they sometimes waited for the project leader's actions. The different ways in which they describe each other makes clear how the tensions they encountered were perceived and explained in different ways. Near the end of the project, Carlo came up with a new version of the syllabus that very strongly expressed the voice of the Commission. Thereby he did not account for all the work done on the previous syllabus, which was labelled as the European Curricular Framework. Although some expressed that they understood the need to satisfy the Commission, some also expressed that they did not understand how Carlo had derived this new format from the previous work done. Whereas others might perceive Carlo's actions as top-down or as advancing a philosophy of the project that the others didn't share, in the light of the description from the Italian project members Carlo's actions simply attempted to steer the project towards a successful ending, accounting 
for the initial project plan. This project plan embodied for them a specific philosophy that related to the situation in their country. The tensions they encountered therefore did not necessarily mean that they did not agree with what the others were aiming for, but solely that different project members saw different things in the project. What they shared is that they all aimed to create something European, expressing a European voice. At the same time they were all searching for something that fitted the local situations of the diverse countries, expressing a national voice. They were also all involved in a project that had to fulfil what had been promised to the European Commission, and they struggled with how to do that. However, they all expressed these voices at different times, and in different ways. Despite the tensions and different understandings they encountered, it remained unexplored in the project what 'philosophy' or rationale lay behind their expressions and arguments about 'the fit with the local situations of their country', about creating something 'European', or about 'the relation with and the aim of the European Commission'. To make decisions often becomes a matter of arguing back and forth, and finding out who gets the last word. In the discussion of the format of the syllabus, the resulting product that was presented as the outcome of the project addressed and reflected most dominantly the voice of the European Commission according to Carlo's understanding. It did not account for the multiple voices brought to the fore during the whole discussion.

\section{Conclusions and Discussion}

The analyses of the threads of discussion showed how multiple voices of the diverse project members can be seen in the negotiation and development of the central conceptual topics. The voices described in the interviews enabled us to see the logic in the arguments they were making and the ways they responded to each other. In contrast, the participants themselves did not explore what the other was aiming for when specific arguments were being made. It remained unclear, therefore, if, how and why there were (mis)understandings or (dis)agreements. The focus of members' discussions was on the project's activities and the products that resolved from it. This seems reasonable given the practical aim and the need to deliver products. However, even when having such an apparent clear and practical aim, our analyses have also shown that conceptual decisions along the way can come to reflect either one dominating voice or a cacaphony of multiple voices. 
Though all project members were aware of certain voices of other project members, they did not explicitly elaborate on those and they described these subjectivities differently. The Danish project members were described by others as being practical, while one Danish project member describes themselves as addressing practitioners and related this to the philosophy of the educational bodies in their country. The Italian project leader Carlo was described by others in terms of using both a top-down and bottom-up approach, and by one as advancing a specific concept of the project not shared by others, whereas an Italian project member described how they as Italians were advancing a specific philosophy that related to the situation in their country.

Referring to our Bakhtinian framework, we could say that the particularity of the diverse voices insufficiently came to the fore in the situated arguments. The broader socio-cultural contexts, the very richness of their diversity, were thus left unexplored by the participants. This is noteworthy as an important motivation of the project members to work together was to learn from each other. There seems to be more, but unexploited, potential for learning during the collaborative work on the practical products, residing in the form of learning about each other's worlds. Especially in those moments where one senses that someone else is expressing a specific subjectivity, coming to the fore most clearly in misunderstandings and disagreements, there is scope to enter into the world of the other. Learning from the expertise of the Danish partners, as one Italian project member hoped for, lies within considering what most project members perceived as the practical comments made by the Danish project members. It was in those comments that the Danish partners were arguing in line with addressing the practitioners, expressing the philosophy of the educational bodies in their country. Similarly, it is in the discussion raised by the Spanish project members about pedagogy that they were expressing their critical view. Again it is these comments that allow one to come to know more about this critical view. The difficulty is, of course, to perceive such situated comments also as expressions of other sociocultural worlds. These comments are most likely the very automized expressions of diverse worlds, and it is likely that neither the one who listens to them, nor the one who makes the comments, is aware of this. So, what is particular about oneself or particular about the other is left untouched as these particularities are not taken or rendered as particularities in the moments that they play a role. Rather, the particularity that lies in the comments of one project member is translated into categories and terms familiar to the project member who listens. The (mis) understandings and (dis)agreements that follow the particular 
comments cannot be considered real responses to these particularities, as the particularities are not what is being addressed in these responses. Referring to studies done by Garfinkel (1967), Gurevitch (1988) noted that 'the understanding of the other is mediated by a grid of familiar typifications; the other as other remains unnoticed' (p. 1183). Such a practice thus effectively eliminates the possibility of encountering diversity. As a result, the clashing of diverse 'cultures of knowing' (Knorr Cetina, 1999) that is argued to be the very potential of these boundary-crossing projects is not realized.

This finding leads us to a crucial aspect for theorizing about the meaning-generative function of boundary crossing. In response to the studies on collaboration that aim to overcome diversity, perceiving it as an obstacle for mutual understanding, on the basis of these findings we want to argue the opposite: diversity should even be enhanced, in the sense that the other should be perceived and treated as an other person. When the project members do not consider what is particular about the arguments made by the other, the world that the other is expressing does not come to fore and therefore does not play a meaning-generating role. In reaction to the dialogical arguments that emphasize that the ambiguity of language and diversity in groups generate meaning, this study has shown that diversity cannot be presupposed (Abbott, 1995). Even when it concerns boundary-crossing groups, involving people from different organizations, nations or disciplines, diversity has to be actively worked on. Meaning to be generated through diversity requires first that the particularities and the possible boundaries between group members become actually visible to them. Concretely, this means that group members should be encouraged to perceive and treat each other as other persons, and to render each other's arguments as strange and new. Such an attitude opens up the possibility to question and learn what other viewpoints are about, although these viewpoints of others always remain to be understood in one's own terms.

Paradoxically, the findings of this boundary-crossing project suggest that for meaning to be generated from diversity, or for the dialogical function to come to work, requires that the other is 'correctly' rendered as an other. The otherness of the other should not be overcome, but should be even augmented. Concretely, this means that we should encourage group members to render each other's arguments as strange and new. Gurevitch (1988) points out that rather than questioning how sameness can be achieved between people, the question for dialogue becomes how to behold the other as other, that is: 'the other as a person who is not understood but nonetheless real and present in the 
perceiver's consciousness as an other' (p. 1184). This is what enables boundary-crossing dialogues to turn into a meaning-generating venture.

\section{Notes}

We express our thanks to the project group that was willing to follow up its collaborative work so intensively. We gratefully acknowledge useful comments on previous versions of this paper by Etienne Wenger, Peter Zomer and by colleagues from the workplace learning group.

1. For reasons of anonymity, the names presented here are pseudonyms.

2. The analysis concerns a complex whole and is informed by the researcher's participant observation as well as by the theoretical perspective of the researcher. It therefore becomes meaningless to check the quality by repeating (parts of) the analysis to see whether it leads to the same results.

3. The audit report written by the auditor about the specifics concerning the trustworthiness of this study can be requested from the first author.

4. We analysed not only the meetings, but also the specific changes that were made in the documents and the comments of those who changed it. Although this in itself raises the question how external representations like the documents mediate communication and collaboration, we did not analyse the processes in that light, leaving this open for future research.

5. The actual descriptions and complete analyses, as well as a more thick description of the nature of the project, can be requested from the first author.

\section{References}

Abbott, A. (1995). Things of boundaries. Social Research, 62, 857-883.

Akkerman, S., Admiraal, W., Brekelmans, M., \& Oost, H. (in press). Auditing quality of research in social sciences. Quality $\mathcal{E}$ Quantity.

Akkerman, S., Admiraal, W., \& Simons, R.J. (2006). Collaborative activities in academic work. Manuscript submitted for publication.

Alexandrov, V.E. (2000). Biology, semiosis, and cultural difference in Lotman's semiosphere. Comparative Literature, 52, 399-423.

Bakhtin, M.M. (1981). The dialogic imagination: Four essays (M. Holquist, Ed. \& Trans.; C. Emerson, Trans.). Austin: University of Texas Press.

Bakhtin, M.M. (1986). Speech genres and other late essays (C. Emerson \& M. Holquist, Eds.; V.W. McGee, Trans.). Austin: University of Texas Press.

Creswell, J.W. (1998). Qualitative inquiry and research design: Choosing among five traditions. Thousand Oaks, CA: Sage.

Davies, B., \& Harré, R. (2001). Positioning: The discursive production of selves. In M. Wetherell, S. Taylor, \& S.J. Yates (Eds.), Discourse theory and practice (pp. 216-271). London: Sage.

Derry, S., Adams DuRussel, L., \& O’Donnell, A.M. (1998). Individual and distributed cognitions in interdisciplinary teamwork: A developing case study and emerging theory. Educational Psychology Review, 10, 25-56. 
Engeström, Y. (1999). Communication, discourse and activity. Communication Review, 3(1-2), 165-185.

Engeström, Y., Engeström, R., \& Kärkäinen, M. (1995). Polycontextuality and boundary crossing in expert cognition: Learning and problem solving in complex work activities. Learning and Instruction, 5, 319-336.

Garfinkel, H. (1967). Studies in ethnomethodology. Englewood Cliffs, NJ: Prentice-Hall.

Geertz, C. (1973). The interpretation of cultures. New York: Basic Books.

Granovetter, M.S. (1973). The strength of weak ties. American Journal of Sociology, 78, 1360-1361.

Guba, E.G. (1981). Criteria for assessing the trustworthiness of naturalisic inquiries. Educational Communication and Technology Journal, 29, 75-91.

Gurevitch, Z.D. (1988). The other side of dialogue: On making the other strange and the experience of otherness. American Journal of Sociology, 93, 1179-1199.

Gutiérrez, K.D., Baquedano-López, P., \& Tejeda, C. (1999). Rethinking diversity: Hybridity and hybrid. Language practices in the third space. Mind, Culture and Activity, 6, 286-303.

Halpern, E.S. (1983). Auditing naturalistic inquiries: The development and application of a model. Unpublished doctoral dissertation, Indiana University, Indiana.

Hermans, H.J.M. (2001). The dialogical self: Toward a theory of personal and cultural positioning. Culture \& Psychology, 7, 243-281.

Hermans, H.J.M., \& Kempen, H.J.G. (1993). The dialogical self: Meaning as movement. San Diego, CA: Academic Press.

Homan, T. (2001). Teamleren. Theorie en facilitatie [Team learning. Theory and facilitation]. Schoonhoven: Academic Services.

Knorr Cetina, K.D. (1999). Epistemic cultures: How the sciences make knowledge. Cambridge, MA: Harvard University Press.

Lotman, Y.M. (1990). Universe of the mind: A semiotic theory of culture (A. Shukman, Trans.). Bloomington: Indiana University Press.

Lotman, Y.M. (1994). Text within a text. Publications of the Modern Language Association, 109, 377-384.

Marks, M.A., Burke, C.S., Sabella, M.J., \& Zaccaro, S.J. (2002). The impact of cross-training on team effectiveness. Journal of Applied Psychology, 87, 3-13.

Mathieu, J.E., Heffner, T.S., Goodwin, G.F., Salas, E., \& Cannon-Bowers, J.A. (2000). The influence of shared mental models on team process and performance. Journal of Applied Psychology, 85, 273-283.

Matusov, E. (1996). Intersubjectivity without agreement. Mind, Culture and Activity, 3, 25-45.

Mohammed, S., \& Ringseis, E. (2001). Cognitive diversity and consensus in group decision making: The role of inputs, processes and outcomes. Organizational Behavior and Human Decision Processes, 85, 310-335.

Morris, P. (Ed.). (1994). The Bakhtin reader. London: Edward Arnold.

Ryan, D.P., Cott, C., \& Robertson, D. (1997). Conceptual tools for thinking about interteam work in clinical gerontology. Educational Gerontology, 23, 651-667. 
Spradley, J.P. (1980). Participant observation. New York: Holt, Rinehart \& Winston.

Star, S.L., \& Griesemer, J.R. (1989). Institutional ecology, 'translations' and boundary objects: Amateurs and professionals in Berkeley's Museum of Vertebrate Zoology. Social Studies of Science, 19, 387-420.

Valsiner, J., \& Van der Veer, R. (2000). The social mind: Construction of the idea. Cambridge: Cambridge University Press.

Wenger, E. (1998). Communities of practice: Learning, meaning and identity. Cambridge: Cambridge University Press.

Wertsch, J.V. (1985). Culture, communication, and cognition: Vygotskian perspectives. New York: Cambridge University Press.

Wertsch, J.V. (1991). Voices of the mind: A sociocultural approach to mediated action. Hemel Hempstead: Harvester Wheatsheaf.

Wertsch, J., \& Toma, C. (1995). Discourse and learning in the classroom: A sociocultural approach. In L.P. Steffle \& J. Gale (Eds.), Constructivism in education (pp. 159-175). Hillsdale, NJ: Erlbaum.

Wetherell, M., Taylor, S., \& Yates, S.J. (2001). Discourse as data. London: Sage.

Yoo, Y., \& Kanawattanachai, P. (2001). Developments of transactive memory systems and collective mind in virtual teams. International Journal of Organizational Analysis, 9, 187-208.

\section{Biographies}

SANNE AKKERMAN successfully defended her thesis on academic collaboration across organizational boundaries in 2006. She has published various scientific papers on the process of collaboration and communication in professional groups. She is now a researcher at the IVLOS Institute of Education of Utrecht University in the Netherlands. ADDRESS: Sanne Akkerman, IVLOS, Utrecht University, PO Box 80127, 3508 TC Utrecht, The Netherlands. [email: s.f.akkerman@ivlos.uu.nl]

WILFRIED ADMIRAAL is head of the Department of ICT and Education at the Graduate School of Teaching and Learning of the University of Amsterdam. His publications cover the use of technology at most levels of education.

ROBERT JAN SIMONS is Director of the Center for ICT in Education of Utrecht University. His list of publications includes papers on educational psychology, workplace learning and the use of ICT in education.

THEO NIESSEN Niessen has been a Ph.D. student at the Faculty of Health Sciences at Maastricht University since 2000. His PhD research focuses on the mediating influence of epistemological beliefs from experienced teachers confronted with reform activities. Since September 2005 he has also been lecturer in Care Ethics at the Professional University of Windesheim. 